\title{
Suppression of ERK/NF-kB Activation Is Associated With Amentoflavone-Inhibited Osteosarcoma Progression In Vivo
}

\author{
YEN-JU LEE ${ }^{1,2}$, JING-GUNG CHUNG $^{3,4}$, YI-TING CHIEN $^{5}$, SONG-SHEI LIN $^{2 *}$ and FEI-TING HSU ${ }^{3 *}$ \\ ${ }^{1}$ Department of Emergency Medicine, Zuoying Branch of Kaohsiung \\ Armed Forces General Hospital, Kaohsiung, Taiwan, R.O.C.; \\ ${ }^{2}$ Department of Medical Imaging and Radiological Sciences, \\ Central-Taiwan University of Science and Technology, Taichung, Taiwan, R.O.C.; \\ ${ }^{3}$ Department of Biological Science and Technology, China Medical University, Taichung, Taiwan, R.O.C.; \\ ${ }^{4}$ Department of Biotechnology, Asia University, Taichung, Taiwan, R.O.C.; \\ ${ }^{5}$ Department of Medical Imaging and Radiology, \\ Shu-Zen Junior College of Medicine and Management, Kaohsiung, Taiwan, R.O.C.
}

\begin{abstract}
Background/Aim: Amentoflavone has been implicated in reducing the metastatic potential of osteosarcoma (OS) cells in vitro. The aim of the present study was to verify the antitumoral efficacy and the potential mechanism of amentoflavone osteosarcoma progression inhibition in vivo. Materials and Methods: A U-2 OS osteosarcoma xenograft mouse model was used in this study. Mice were treated with a vehicle control or amentoflavone (100 $\mathrm{mg} / \mathrm{kg} /$ day) for 15 days. Tumor growth, signal transduction, and expression of tumor progression-associated proteins were evaluated using a digital caliper, bioluminescence imaging (BLI), animal computed tomography $(C T)$, and ex vivo western blotting assay. Results: Amentoflavone significantly inhibits tumor growth and reduces protein levels of phospho-extracellular signal-regulated kinase $(P-E R K)$, nuclear factor-kappaB $(N F-\mathrm{k} B) \quad p 65$ (Ser536), vascular endothelial growth factor (VEGF), matrix metallopeptidase 9 (MMP-9), X-linked inhibitor of apoptosis protein (XIAP), and cyclin-D1 in osteosarcoma in vivo. Conclusion: The inhibition of ERK/NF- $\mathrm{K} B$ activation is associated with amentoflavone-inhibited osteosarcoma progression in vivo.
\end{abstract}

\footnotetext{
*These Authors contributed equally to this study.

Correspondence to: Song-Shei Lin, Department of Medical Imaging and Radiological Sciences, Central Taiwan University of Science and Technology, Taichung, Taiwan, R.O.C. Tel: +886-4-22391647-7111, Email: sslin@ctust.edu.tw and Fei-Ting Hsu, Department of Biological Science and Technology, China Medical University, No 91, Hsueh-Shih Road, Taichung, Taiwan 404, R.O.C. Tel: +886 422053366 (ext. 2532), Fax: +886 422053764, e-mail: sakiro920@gmail.com
}

Key Words: Osteosarcoma, amentoflavone, ERK, NF-кB.
Herbal medicines are being used for the maintenance of healthy bones and for treating bone-related diseases for thousands of years (1). Many herbal compounds and composite formulas have been shown to prevent osteoporosis and relief bone pain by upregulating the natural osteoprotective and anti-inflammatory responses of the body $(1,2)$. The multi-herb formula, Er-Zhi-Wan, has been implicated in the prevention of osteoporosis by suppressing osteoclastic bone resorption (3). It was reported that Xiao$\mathrm{Ai}$-Tong (one of the traditional Chinese medicine) can reduce the bone cancer related pain of patient via the modulation of cytokine and against adverse reaction of morphine (4).

In addition, the antitumoral effect of herbal medicine in osteosarcoma has been reported by Chang et al., who have summarized and evaluated the therapeutic efficacy of herbal medicines against osteosarcoma growth in mice using a systematic review and meta-analysis (5). Specific herbal compounds have been recognized as apoptotic inducers of osteosarcoma suppressing tumor cell growth (6), such as curcumin, a bioactive compound isolated from Curcuma longa, which can inhibit osteosarcoma cell proliferation by triggering apoptosis (7).

Several intracellular signaling pathways that participate in tumor growth are activated through the overexpression of signaling kinases and correlate with a poor outcome in osteosarcoma (8). As a consequence, specific kinase inhibitors have been generated to abolish osteosarcoma growth through the suppression of several signal transduction pathways (9, 10). Pre-clinical data have demonstrated that phosphoinositide 3-kinase (PI3K) and the mammalian target of rapamycin (mTOR) inhibitors can prevent cell growth and induce cell cycle arrest and apoptosis in osteosarcoma cell lines (10). Clinical trials have shown that multi-kinase inhibitors, such as sorafenib and regorafenib, can prolong progression-free 
survival in patients with high-grade osteosarcoma following failure of conventional therapy $(11,12)$.

Herbal compounds have been documented to effectively inhibit osteosarcoma cell proliferation, survival, and invasion, through inactivation of signaling kinases. Formononetin, a component of Radix astragali, induces cell apoptosis by inactivating extracellular signal-regulated kinase (ERK) and protein kinase B (AKT) in osteosarcoma (13). Amentoflavone, a multifunctional compound extracted from Selaginella tamariscina, has also been shown to downregulate the metastatic potential of osteosarcoma in vitro by suppressing ERK activation (14). In our previous studies, we found that amentoflavone inhibits tumor growth by suppressing ERK in hepatocellular carcinoma both in vitro and in vivo $(15,16)$. However, the antitumoral effect and mechanism of action of amentoflavone in osteosarcoma in vivo is not clear. The aim of the present study was to evaluate the antitumoral efficacy and potential mechanism of amentoflavone's action on osteosarcoma in vivo.

\section{Materials and Methods}

Chemical reagents and antibodies. Amentoflavone was purchased from Sigma-Aldrich (St. Louis, MO, USA) and was dissolved in dimethyl sulfoxide (DMSO, from Sigma-Aldrich). pGL4.50 (luc2/CMV) vector and D-luciferin were acquired from Promega (Madison, WI, USA). JetPEI ${ }^{\mathrm{TM}}$ transfection reagent was purchased from Polyplus Transfection (S'elestat, Bas-Rhin, France). Primary and secondary antibodies involving anti-VEGF (EMD Millipore Corporation, Burlington, MA, USA), anti-MMP-9 antibody (EMD Millipore Corporation), anti-XIAP (Thermo Fisher Scientific, Fremont, CA, USA), anti-ERK1/2 antibody clone MK12 (Santa Cruz Biotechnology, Inc. Dallas, TX, USA), antiphospho-ERK1/2 antibody (Thr202/Tyr204, Thr185/Tyr187, Cell Signaling Technology, Inc., Danvers, MA, USA), anti-mouse IgG and anti-rabbit IgG (Jackson ImmunoResearch,West Grove, PA, USA) were all used in Western blot assays

U-2 OS cell line. Human osteosarcoma U-2 OS cells were generously provided by Professor Jing-Gung Chung from the Department of Biological Science and Technology, China Medical University, Taichung, Taiwan. McCoy's 5A medium culture media contained 10\% FBS, 2 mM L-glutamine, and PS (100 Units $/ \mathrm{ml}$ and $100 \mu \mathrm{g} / \mathrm{ml}$ ) (Life Technologies, Carlsbad, CA, USA). U-2 OS cells were cultured at $80 \%$ confluence and were then maintained at $37^{\circ} \mathrm{C}$ in a humidified incubator with $5 \% \mathrm{CO}_{2}$ atmosphere $(14,17)$.

Plasmid transfection and stable clone selection. U-2 OS cells were seeded as $80 \%$ confluence in a $6-\mathrm{cm}$ plate before pGL4.50 luciferase reporter [pGL4.50 (luc2/CMV)] vector transfection. The transfection protocol was followed by the JetPEI ${ }^{\mathrm{TM}}$ transfection kit (S'elestat, Bas-Rhin, France), according to the manufacturer's instructions. Following transfection, hygromycin $(100 \mu \mathrm{g} / \mathrm{ml})$ (Santa Cruz Biotechnology, Santa Cruz, CA, USA) was added for the selection and stabilization of the luciferase-expressing cells. Finally, U-2 OS cells with a stable luciferase expression were identified by a bioluminescence imaging system (Xtreme, Bruker, Billerica, MA, USA) and defined as U-2 OS/luc2 cells $(14,18)$.
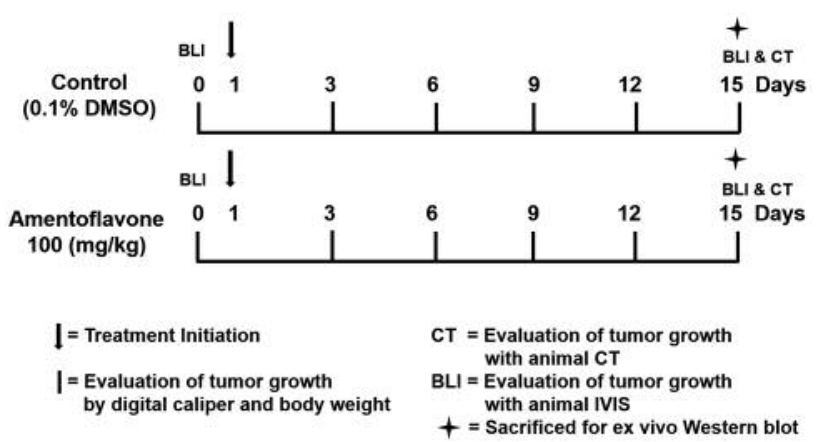

Figure 1. Animal experiment procedure. Nude mice bearing $U-2$ OS/luc2 cells were separated into control and amentoflavone-treated groups. Tumor size and weight and whole-body weight were evaluated every 3 days by caliper and scale, respectively. Bioluminescence imaging for each group was acquired before treatment (day 0) and on day 15. CT scan was performed on day 15.

$U$-2OS xenograft animal model. The animal study was approved by the Institutional Animal Care and Use Committee in Taipei Medical University (certification number: LAC-2017-0223). All animal procedures were followed by the guide for the care and use of laboratory animals under the 5R's and 5F's principles (19). Sixweek-old male nude $(n u / n u)$ mice were purchased from the National Laboratory Animal Center (Taipei, Taiwan). For the xenograft animal model, U-2 OS/luc 2 cells $\left(1 \times 10^{7}\right.$ in $200 \mu \mathrm{L}$ PBS $)$ were injected subcutaneously at the left leg of the nude mice $(20,21)$. The animal experimental procedure is represented in Figure 1. When tumor size reached $100 \mathrm{~mm}^{3}$, the mice were randomly separated into two groups ( $\mathrm{N}=5 / \mathrm{per}$ group). The control group was treated with $100 \mu \mathrm{L}$ PBS with $0.1 \%$ DMSO by gavage. Another group was administered with amentoflavone $(100 \mathrm{mg} / \mathrm{kg})$ dissolved in $100 \mu \mathrm{L}$ PBS with $0.1 \%$ DMSO. Treatment began from day 1 until day 15 (a total of fifteenth days of treatment). The tumors' volume and mice whole body weight were measured every 3 days, and the tumor volume was calculated using the formula $V=$ width $\times$ length $\times$ height $\times 0.523$. Mice were sacrificed on day 15 and tumor specimens were collected for ex vivo Western blotting analyses.

In vivo bioluminescence imaging (BLI). In vivo luminescence images of mice bearing U-2 OS/luc2 cells were taken on days 0 (before treatment) and 15 (after fifteen daily treatments) (Xtreme, Bruker, Billerica, MA, USA). Mice were intraperitoneally injected with $200 \mu \mathrm{L}$ D-luciferase $(15 \mathrm{mg} / \mathrm{kg})$ for $15 \mathrm{~min}$ and were anesthetized by $1-3 \%$ isoflurane. Isoflurane gas anesthesia was maintained at $1.5 \%$ during the 3-minute acquisition of the luciferase signal from tumor. Photon signal accumulated from the tumor in each mouse was quantified using Molecular Imaging Software (MI) version 7.2 (Bruker) (18).

In vivo whole-body computer tomography. Nude mice bearing U-2 OS/luc2 cells were anesthetized by $1-3 \%$ isoflurane and were prepared for scanning using computer tomography (Mediso Ltd., Budapest, Hungary) on day 15 (after fifteen daily treatments) with specific operation parameters: i) tube energy $55 \mathrm{kVp} \times 145 \mu \mathrm{A}$, ii) 360 projections, iii) voxel size $145 \times 145 \times 145 \mu \mathrm{M}$ (16). After 
A

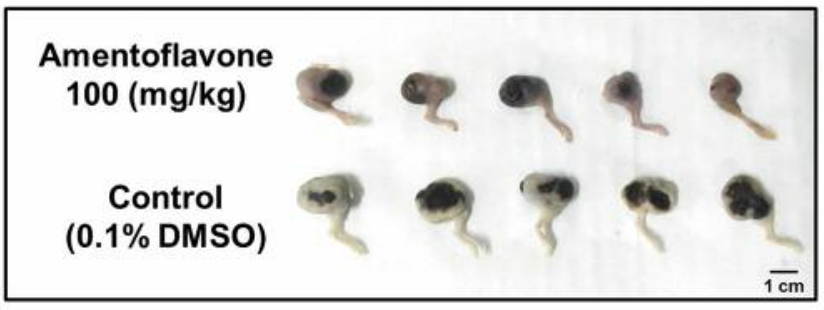

B

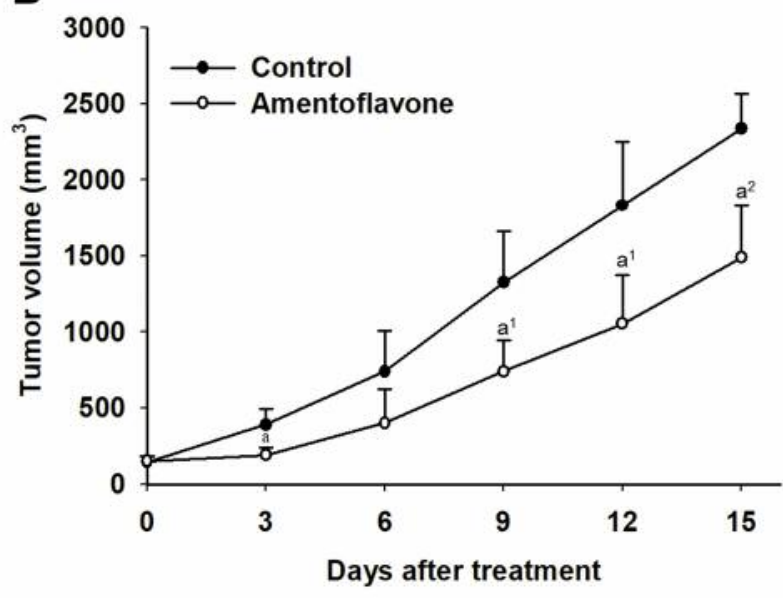

D

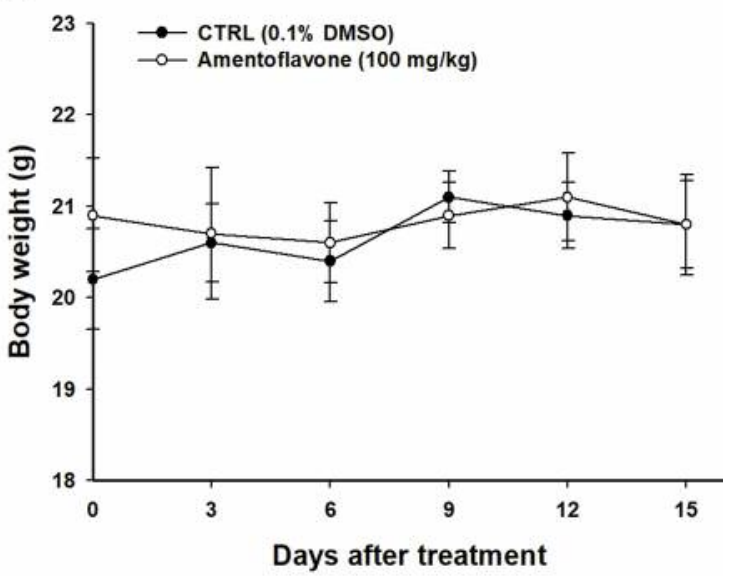

Figure 2. Tumor growth was suppressed by amentoflavone without inducing general toxicity. (A) Tumor tissue images from controls and amentoflavone-treated mice. (B) Quantified result of tumor size, (C) tumor weight, and (D) body weight from each group. $\left(a^{1}: p<0.05, a^{2}: p<0.01\right.$ vs. control).

scanning, mice were sacrificed on the same day (day 15) for protein analysis.

Ex vivo Western blot. Ex vivo Western blotting was performed as previously described (18). Briefly, tumor tissue was pulverized using sonication, and was solubilized using nuclear and cytoplasmic extraction kits (EMD Millipore). Proteins were subjected to SDS PAGE gel electrophoresis and were transferred to a polyvinylidene difluoride membrane (Thermo Fisher Scientific). Membranes were blocked with $3 \%$ bovine serum albumin for 1 hour and were incubated overnight with the respective primary antibodies. Membranes were then washed 3 times with TBST (Tris-buffered saline and $0.1 \%$ Tween 20; Sigma-Aldrich, St. Louis, MO, USA) and were incubated with the secondary antibody $(1: 10,000)$ for another 1 hour. Relative protein expression bands were visualized using enhanced chemi-luminescence (Thermo Fisher Scientific) and were detected using the ChemiDoc MP Imaging System (Bio-Rad Laboratories, Inc., CA, USA) (22).

\section{Results}

Amentoflavone may effectively suppress $U-2$ OS/luc2 tumor growth without general toxicity. The animal experimental flow chart is shown in Figure 1. Tumors isolated from each treatment group are shown in Figure 2A. The caliper measurements (Figure 2B) indicate a marked decrease of tumor growth following amentoflavone treatment. The average tumor tissue weight in the amentoflavone-treated group on day 15 was also reduced as compared to control group (Figure 2C). To evaluate the general toxicity of the treatment we also recorded the body weight of mice during the therapy. The body weight of control and amentoflavone-treated mice was maintained around 20-21 $\mathrm{g}$ or both groups from the start of the treatment till the end (Figure 2D). 
A

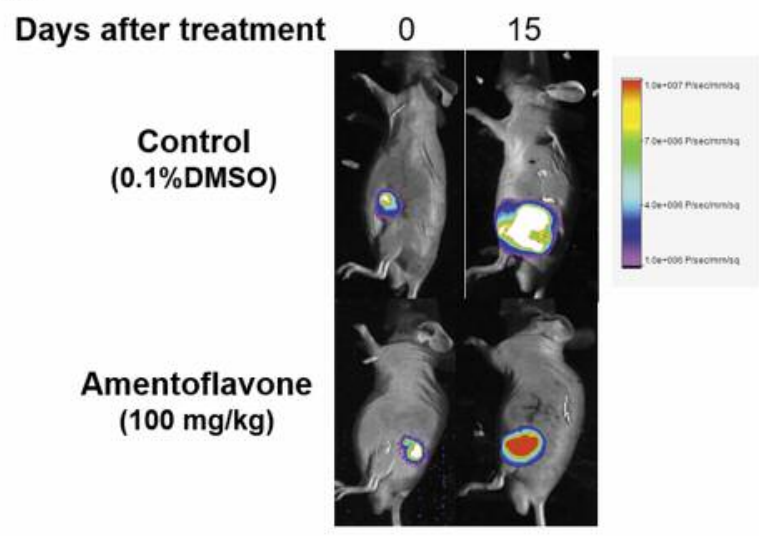

C

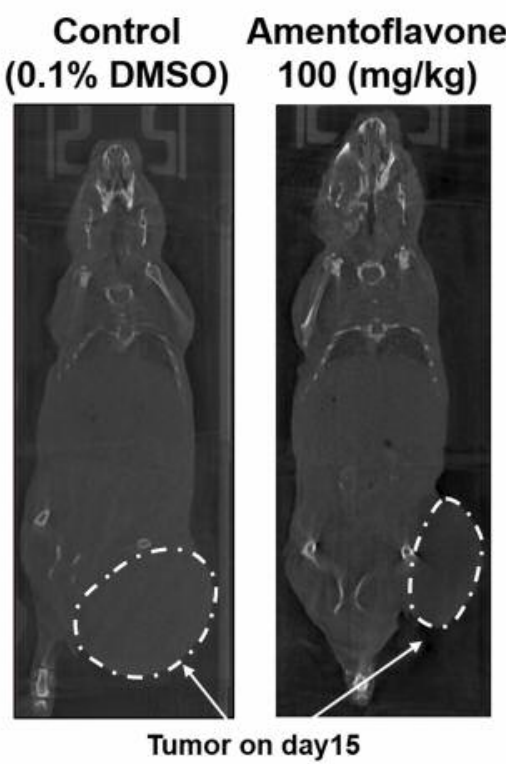

Both BLI and CT imaging showed marked tumor growth suppression following amentoflavone treatment. In order to confirm the effect of amentoflavone, we evaluated tumor growth by two different imaging methods. BLI images of one representative mouse from each group on days 0 (before treatment) and 15 (after fifteen daily treatments) are shown in Figure 3A. The quantified result of the total photon signal from the tumor area showed signal drop only in the amentoflavone treatment group during the same time (Figure 3B). In addition, whole body CT imaging on the same day showed that tumor size was effectively reduced in amentoflavone-treated mice compared to control (Figure 3C).

Tumor progression effect was inhibited by amentoflavone treatment via ERK/NF-kB signaling inactivation. To
B

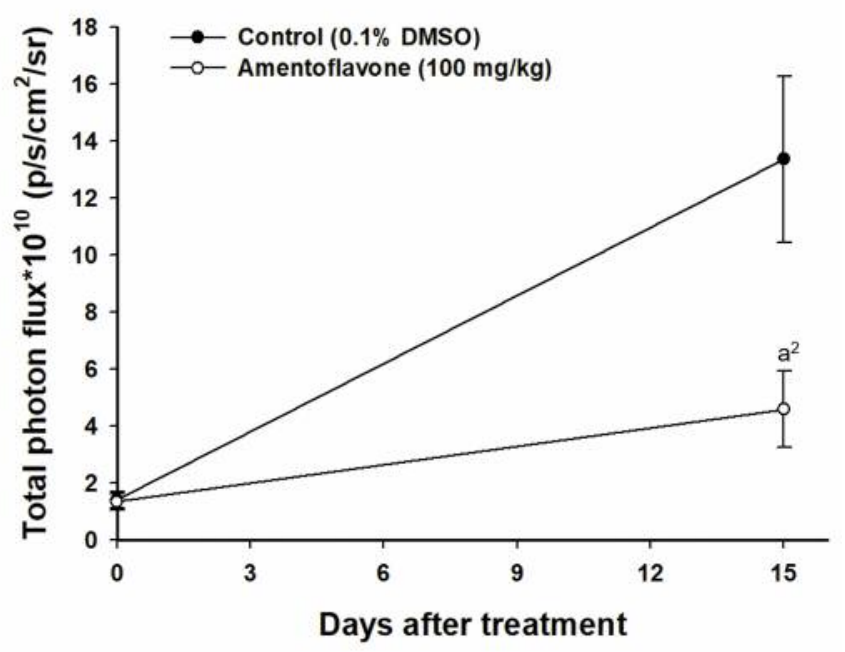

Figure 3. BLI and CT images indicate that the tumor size decreases in the amentoflavone-treated mice. (A) Bioluminescence images of one representative mouse from each group were displayed. (B) Photon intensity from each mouse tumor area was measured and quantified. (C) White dot line points out the tumor area on whole body CT images. $\left(a^{2}\right.$ : $p<0.01$ vs. control).

investigate the molecular mechanism of amentoflavone of osteosarcoma growth inhibition, we evaluated the expression of ERK/NF-KB and their downstream regulators in control and amentoflavone-treated tumor tissues. In figures $4 \mathrm{~A}$ and $\mathrm{B}$, the phosphorylation of P65 NF-kB and P-ERK were markedly decreased following amentoflavone treatment. Furthermore, NF-kB downstream effector proteins, including VEGF, MMP9, XIAP (angiogenesis, invasion and migration related proteins, respectively) and CyclinD1 (proliferation related protein) were all suppressed following amentoflavone treatment $(14,30,31)$ (Figures 4C and D). Collectively, we saw that amentoflavone effectively suppresses osteosarcoma progression via inhibition of ERK/NF-kB signaling pathways. 

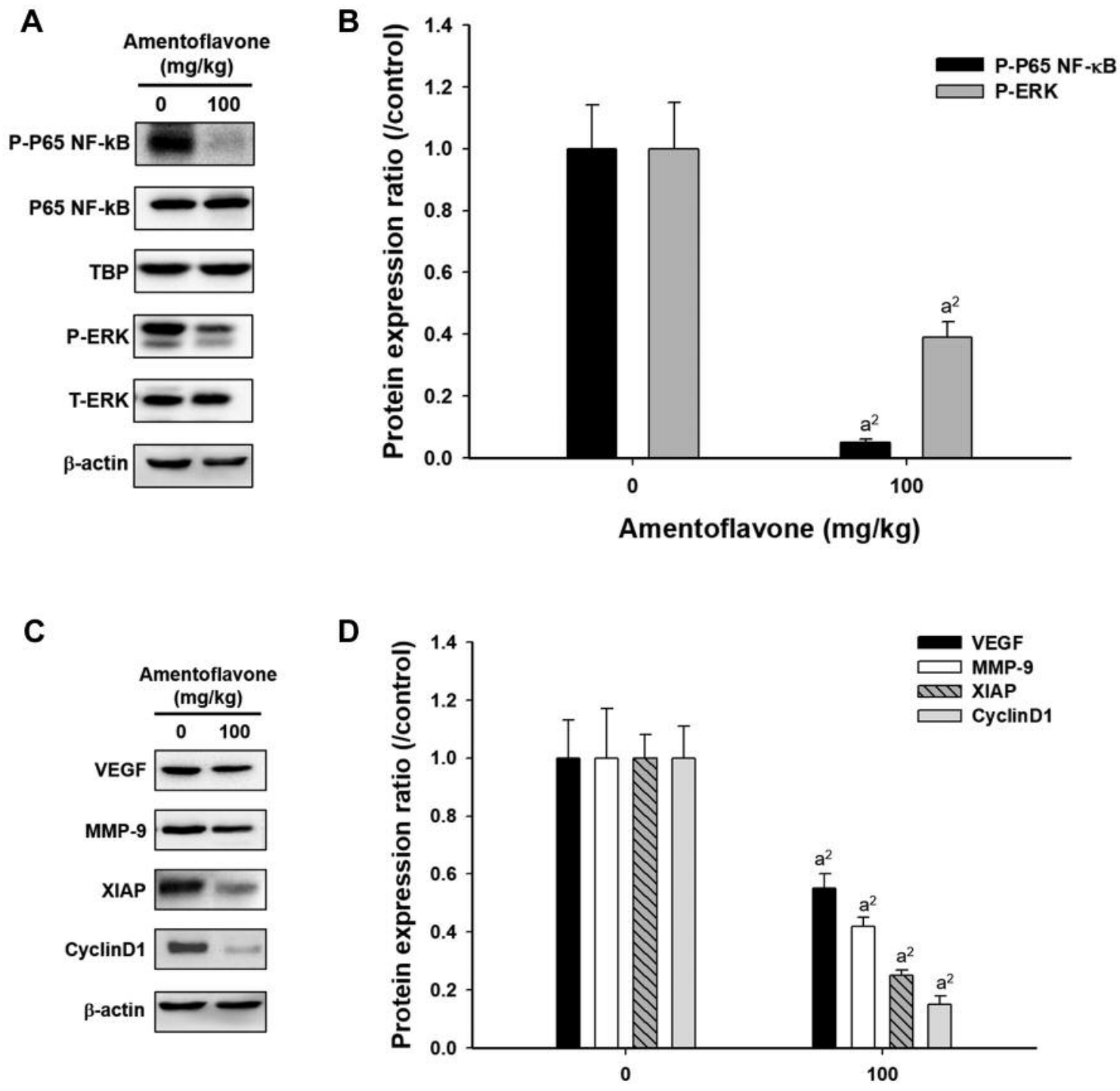

Amentoflavone $(\mathrm{mg} / \mathrm{kg})$

Figure 4. Tumor progression-related proteins decrease by amentoflavone. (A, C) Ex vivo Western blots and (B, D) quantified bar charts of phosphop65- NF-кB, phospho-ERK, VEGF, MMP9, XIAP and CyclinD1. $\left(a^{2}: p<0.01\right.$ vs. control $)$.

\section{Discussion}

The rapid progression of metastatic osteosarcoma diminishes the efficacy of conventional therapy and results in poor survival (23). Tumor progression-associated proteins such as VEGF, MMP-9, XIAP, and Cyclin-D1 have been shown to control tumor angiogenesis, invasion, anti-apoptosis, and proliferation in osteosarcoma. Inhibition of these proteins with small interfering- or micro-RNA (siRNA or miRNA) not only has previously reduced osteosarcoma tumor progression but has also enhanced the efficacy of therapeutic agents (24-27).
The NF-kB p50/p65 heterodimer plays the role of a critical modulator in tumor development and progression and numerous tumor progression-associated proteins are encoded by NF-kB target genes. For example, NF-kB activation modulates the resistance of tumors to chemotherapeutic agents and radiation, while inhibition of NF-kB signaling may enhance treatment efficacy $(28,29)$. The increased NF-kB nuclear translocation ss associated with poor survival in patients with osteosarcoma (30), while protein levels of VEGF, MMP-9, XIAP, and Cyclin-D1 are reduce upon NF-kB inactivation in osteosarcoma $(14,30,31)$. 
The phosphorylation of Mitogen-Activated Protein Kinases (MAPK)/ERK mediate oncogenic phenotypes, including cell growth, survival, invasion, and angiogenesis by triggering the activation of downstream transcription factors in cancer cells $(32,33)$. Increased P-ERK expression has also been linked to chemoresistance in patients with osteosarcoma (34), while PD98059, an ERK inhibitor, can suppress NF-kB activation and reduce the expression of metastasis-associated proteins in osteosarcoma U2-OS cells in vitro (14).

ERK phosphorylation has been considered as a therapeutic target for osteosarcoma $(9,32)$. Sorafenib, a multi-kinase inhibitor, has been shown to induce ERK dephosphorylation in osteosarcoma in vitro and works as a second- or third-line treatment to improve the progression-free survival of patients with high-grade osteosarcoma following failure of multimodal chemotherapy $(9,11,32)$. In fact, our own data demonstrated that amentoflavone significantly reduces tumor growth as well as the expression of P-ERK, NF-kB p65 (Ser536), and other osteosarcoma tumor progressionassociated proteins in vivo.

In conclusion, the suppression of ERK/NF- $\mathrm{KB}$ signaling is associated with amentoflavone-inhibited osteosarcoma progression in vivo. Further clinical trials need to be arranged and validated in the future work before clinical application. Here, we suggest that amentoflavone may be a potential adjuvant therapy for the treatment of osteosarcoma.

\section{Conflicts of Interest}

The Authors declare no conflicts of interest.

\section{Authors' Contributions}

Data curation was performed by YJL and FTH, the writing and original draft preparation as done by YJL, YTC and FTH. All Authors contributed to writing, and supervision of the study was done by JGC and SSL.

\section{Acknowledgements}

This study was supported by Zuoying Branch of Kaohsiung Armed Forces General Hospital, Kaohsiung, Taiwan, R.O.C (ID: ZBH 107-11). Experiments and data analysis were performed in part through the use of the Medical Research Core Facilities, Office of Research 2 Development at China Medical University, Taichung, Taiwan, R.O.C.

\section{References}

1 Mukwaya E, Xu F, Wong MS and Zhang Y: Chinese herbal medicine for bone health. Pharm Biol 52: 1223-1228, 2014. PMID: 24963946. DOI: 10.3109/13880209.2014.884606

2 Mobasheri A: Intersection of inflammation and herbal medicine in the treatment of osteoarthritis. Curr Rheumatol Rep 14: 604616, 2012. PMID: 22987043. DOI: 10.1007/s11926-012-0288-9
3 Qin L, Han T, Zhang Q, Cao D, Nian H, Rahman K and Zheng $\mathrm{H}$ : Antiosteoporotic chemical constituents from er-xian decoction, a traditional chinese herbal formula. J Ethnopharmacol 118: 271279, 2008. PMID: 18501540. DOI: 10.1016/j.jep.2008.04.009

4 Cong Y, Sun K, He X, Li J, Dong Y, Zheng B, Tan X and Song $\mathrm{X}-\mathrm{J}$ : A traditional chinese medicine xiao-ai-tong suppresses pain through modulation of cytokines and prevents adverse reactions of morphine treatment in bone cancer pain patients. Mediators Inflamm 2015: 961635-961635, 2015. PMID: 26617438. DOI: $10.1155 / 2015 / 961635$

5 Chang JL, Wang WY, Li YM, Hu SP, Yao M, Cui XJ, Zhi WL, Shi Q, Wang YJ and Yang YP: Chinese herbal medicine for osteosarcoma in the mouse: A systematic review and metaanalysis. Chin J Integr Med, 2018. PMID: 30484018. DOI: 10.1007/s11655-018-2565-6

6 Li J, Yang Z, Li Y, Xia J, Li D, Li H, Ren M, Liao Y, Yu S, Chen $\mathrm{Y}$, Yang $\mathrm{Y}$ and Zhang $\mathrm{Y}$ : Cell apoptosis, autophagy and necroptosis in osteosarcoma treatment. Oncotarget 7(28): 4476344778, 2016. PMID: 27007056. DOI: 10.18632/oncotarget.8206

7 Jin S, Xu HG, Shen JN, Chen XW, Wang H and Zhou JG: Apoptotic effects of curcumin on human osteosarcoma u2os cells. Orthop Surg 1: 144-152, 2009. PMID: 22009832. DOI: 10.1111/j.1757-7861.2009.00019.x

8 Kansara M, Teng MW, Smyth MJ and Thomas DM: Translational biology of osteosarcoma. Nat Rev Cancer 14: 722735, 2014. PMID: 25319867. DOI: $10.1038 / \operatorname{nrc} 3838$

9 Pignochino Y, Grignani G, Cavalloni G, Motta M, Tapparo M, Bruno S, Bottos A, Gammaitoni L, Migliardi G, Camussi G, Alberghini M, Torchio B, Ferrari S, Bussolino F, Fagioli F, Picci $\mathrm{P}$ and Aglietta M: Sorafenib blocks tumour growth, angiogenesis and metastatic potential in preclinical models of osteosarcoma through a mechanism potentially involving the inhibition of erk1/2, mcl-1 and ezrin pathways. Mol Cancer 8, 2009. PMID: 20003259. DOI: $10.1186 / 1476-4598-8-118$

10 Bishop MW and Janeway KA: Emerging concepts for pi3k/mtor inhibition as a potential treatment for osteosarcoma. F1000Res 5, 2016. PMID: 27441088. DOI: 10.12688/f1000research.8228.1

11 Grignani G, Palmerini E, Dileo P, Asaftei SD, D'Ambrosio L, Pignochino Y, Mercuri M, Picci P, Fagioli F, Casali PG, Ferrari $\mathrm{S}$ and Aglietta M: A phase ii trial of sorafenib in relapsed and unresectable high-grade osteosarcoma after failure of standard multimodal therapy: An italian sarcoma group study. Ann Oncol 23: 508-516, 2012. PMID: 21527590. DOI: 10.1093/ annonc/mdr 151

12 Duffaud F, Mir O, Boudou-Rouquette P, Piperno-Neumann S, Penel N, Bompas E, Delcambre C, Kalbacher E, Italiano A, Collard O, Chevreau C, Saada E, Isambert N, Delaye J, Schiffler C, Bouvier C, Vidal V, Chabaud S and Blay JY: Efficacy and safety of regorafenib in adult patients with metastatic osteosarcoma: A non-comparative, randomised, double-blind, placebo-controlled, phase 2 study. Lancet Oncol 20: 120-133, 2019. PMID: 30477937. DOI: 10.1016/S1470-2045(18)30742-3

13 Liu Y, He J, Chen X, Li J, Shen M, Yu W, Yang Y and Xiao Z: The proapoptotic effect of formononetin in human osteosarcoma cells: Involvement of inactivation of erk and akt pathways. Cell Physiol Biochem 34: 637-645, 2014. PMID: 25170541. DOI: 10.1159/000363029

14 Pan PJ, Tsai JJ and Liu YC: Amentoflavone inhibits metastatic potential through suppression of erk/nf-kappab activation in 
osteosarcoma u2os cells. Anticancer Res 37(9): 4911-4918, 2017. PMID: 28870912. DOI: 10.21873/anticanres.11900

15 Lee KC, Tsai JJ, Tseng C-W, Kuo YC, Chuang YC, Lin SS and Hsu FT: Amentoflavone inhibits erk-modulated tumor progression in hepatocellular carcinoma in vitro. In Vivo 32: 549-554, 2018. PMID: 29695559. DOI: 10.21873/invivo.112274

16 Lee KC, Chen WT, Liu YC, Lin SS and Hsu FT: Amentoflavone inhibits hepatocellular carcinoma progression through blockage of erk/nf-kb activation. In Vivo 32: 1097-1103, 2018. PMID: 30150431. DOI: 10.21873 /invivo.11351

17 Liao CL, Lai KC, Huang AC, Yang JS, Lin JJ, Wu SH, Gibson Wood W, Lin JG and Chung JG: Gallic acid inhibits migration and invasion in human osteosarcoma u- 2 os cells through suppressing the matrix metalloproteinase- $2 /-9$, protein kinase $b$ (pkb) and pkc signaling pathways. Food Chem Toxicol 50: 17341740, 2012. PMID: 22387266. DOI: 10.1016/j.fct.2012.02.033

18 Weng MC, Wang MH, Tsai JJ, Kuo YC, Liu YC, Hsu FT and Wang HE: Regorafenib inhibits tumor progression through suppression of erk/nf-kappab activation in hepatocellular carcinoma bearing mice. Biosci Rep 38(3), 2018. PMID: 29535278. DOI: $10.1042 / B S R 20171264$

19 Kumar AHS: Effectively communicating the 5r's (replace, reduce, refine, reuse, and rehabilitate) of research ethics, biomedical waste, personalized medicines and the rest. J Nat Sc Biol Med 3: 1-2, 2012. PMID: 22690043. DOI: 10.4103/09769668.95932

20 Tang Q-L, Xie X-B, Wang J, Chen Q, Han A-J, Zou C-Y, Yin JQ, Liu D-W, Liang Y, Zhao Z-Q, Yong B-C, Zhang R-H, Feng Q-S, Deng W-G, Zhu X-F, Zhou BP, Zeng Y-X, Shen J-N and Kang T: Glycogen synthase kinase- $3 \beta$, nf-kb signaling, and tumorigenesis of human osteosarcoma. J Natl Cancer Inst 104: 749-763, 2012. PMID: 22534782. DOI: 10.1093/jnci/djs210

21 Liao D, Zhong L, Duan T, Zhang RH, Wang X, Wang G, Hu K, Lv X and Kang T: Aspirin suppresses the growth and metastasis of osteosarcoma through the nf-kappab pathway. Clin Cancer Res 21: 5349-5359, 2015. PMID: 26202947. DOI: 10.1158/ 1078-0432.CCR-15-0198

22 Samkoe KS, Tichauer KM, Gunn JR, Wells WA, Hasan T and Pogue BW: Quantitative in vivo immunohistochemistry of epidermal growth factor receptor using a receptor concentration imaging approach. Cancer Res 74: 7465-7474, 2014. PMID: 25344226. DOI: 10.1158/0008-5472.CAN-14-0141

23 Ando K, Heymann M-F, Stresing V, Mori K, Rédini F and Heymann D: Current therapeutic strategies and novel approaches in osteosarcoma. Cancers 5: 591-616, 2013. PMID: 24216993. DOI: $10.3390 /$ cancers 5020591

24 Peng N, Gao S, Guo X, Wang G, Cheng C, Li M and Liu K: Silencing of vegf inhibits human osteosarcoma angiogenesis and promotes cell apoptosis via vegf/pi3k/akt signaling pathway. Am J Transl Res 8: 1005-1015, 2016. PMID: 27158386.

25 Tian $X$ and Zhang X: A single nucleotide polymorphism (rs 1056629) in 3'-utr of mmp-9 is responsible for a decreased risk of metastatic osteosarcoma by compromising its interaction with microrna-491-5p. Cell Physiol Biochem 38: 1415-1424, 2016. PMID: 27023472. DOI: $10.1159 / 000443084$

$26 \mathrm{Qu} \mathrm{Y,} \mathrm{Xia} \mathrm{P,} \mathrm{Zhang} \mathrm{S,} \mathrm{Pan} \mathrm{S} \mathrm{and} \mathrm{Zhao} \mathrm{J:} \mathrm{Silencing} \mathrm{xiap}$ suppresses osteosarcoma cell growth, and enhances the sensitivity of osteosarcoma cells to doxorubicin and cisplatin. Oncol Rep 33: 1177-1184, 2015. PMID: 25572427. DOI: 10.3892/or.2014.3698

27 Yu X, Pang L, Yang T and Liu P: Lncrna linc01296 regulates the proliferation, metastasis and cell cycle of osteosarcoma through cyclin d1. Oncol Rep 40: 2507-2514, 2018. PMID: 30226542. DOI: $10.3892 /$ or.2018.6674

28 Pacifico F and Leonardi A: Nf-kappab in solid tumors. Biochem Pharmacol 72: 1142-1152, 2006. PMID: 16956585. DOI: 10.1016/j.bcp.2006.07.032

29 Liu YC, Chiang IT, Hsu FT and Hwang JJ: Using nf-kappab as a molecular target for theranostics in radiation oncology research. Expert Rev Mol Diagn 12(2): 139-146, 2012. PMID: 22369374. DOI: $10.1586 / \mathrm{erm} .12 .2$

30 Tang QL, Xie XB, Wang J, Chen Q, Han AJ, Zou CY, Yin JQ, Liu DW, Liang Y, Zhao ZQ, Yong BC, Zhang RH, Feng QS, Deng WG, Zhu XF, Zhou BP, Zeng YX, Shen JN and Kang T: Glycogen synthase kinase-3beta, nf-kappab signaling, and tumorigenesis of human osteosarcoma. J Natl Cancer Inst 104: 749-763, 2012. PMID: 22534782. DOI: $10.1093 /$ jnci/djs210

31 Wang Y, Fu Q and Zhao W: Tetramethylpyrazine inhibits osteosarcoma cell proliferation via downregulation of nf-kappab in vitro and in vivo. Mol Med Rep 8: 984-988, 2013. PMID: 23912183. DOI: $10.3892 / \mathrm{mmr} .2013 .1611$

32 Chandhanayingyong C, Kim Y, Staples JR, Hahn C and Lee FY: Mapk/erk signaling in osteosarcomas, ewing sarcomas and chondrosarcomas: Therapeutic implications and future directions. Sarcoma 2012: 404810-404810, 2012. PMID: 22577336. DOI: $10.1155 / 2012 / 404810$

33 Sever R and Brugge JS: Signal transduction in cancer. Cold Spring Harb Perspect Med 5, 2015. PMID: 25833940. DOI: 10.1101/cshperspect.a006098

34 Salas S, Jiguet-Jiglaire C, Campion L, Bartoli C, Frassineti F, Deville J-L, Maues De Paula A, Forest F, Jézéquel P, Gentet J$\mathrm{C}$ and Bouvier C: Correlation between erk 1 and stat 3 expression and chemoresistance in patients with conventional osteosarcoma. BMC cancer 14: 606-606, 2014. PMID: 25146150. DOI: 10.1186/1471-2407-14-606
Received March 12, 2019

Revised April 8, 2019

Accepted April 10, 2019 